\title{
Infliximab therapy provides beneficial effects for choroidal thickness increase in patients with active ankylosing spondylitis: A possible mechanism mediating the suppressing of uveitis attacks
}

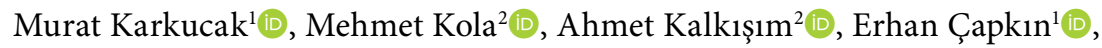 \\ Ahmet Ayar² ${ }^{3}$, Adem Türk² \\ ${ }^{1}$ Department of Orthopedics and Traumatology, Karadeniz Technical University Medical School, Trabzon, Turkey \\ ${ }^{2}$ Department of Ophthalmology, Karadeniz Technical University Medical School, Trabzon, Turkey \\ ${ }^{3}$ Department of Physiology, Karadeniz Technical University Medical School, Trabzon, Turkey
}

\begin{abstract}
Objectives: This study aims to quantitatively assess the profile of the choroidal thickness (ChT) in patients with ankylosing spondylitis (AS) using optical coherence tomography (OCT), and to examine whether the posterior eye segment abnormalities in active AS patients are reversible by infliximab therapy.

Patients and methods: Thirty-one patients with AS ( 22 males, 9 females; mean age: $39.6 \pm 12.3$ years; range, 22 to 68 years) and 24 healthy controls (16 males, 8 females; mean age: $40.8 \pm 8.9$ years; range, 35 to 61 years) were enrolled between October 2014 and March 2016. Patients' clinical and demographic characteristics were recorded. Using OCT, we performed retinal nerve fiber layer (RNFL) thickness, ganglion cell complex, and ChT measurements in AS patients before and six months after the initiation of infliximab therapy, and in healthy controls.

Results: At baseline, patients with AS had higher ChT (mean \pm standard deviation: $347.5 \pm 114.4 \mu \mathrm{m})$ compared to healthy controls $(322.1 \pm 62.8 \mu \mathrm{m})$, although this did not reach statistical significance level $(p=0.283)$. At six months after the first measurement, the mean ChT was significantly decreased (under infliximab therapy: $326.5 \pm 99.7 \mu \mathrm{m}$ vs. before: $347.5 \pm 114.4 \mu \mathrm{m}, \mathrm{p}=0.018$ ) in AS group, while no significant change was observed in the control group ( $p=0.102)$. RNFL thickness in the AS group was significantly decreased after six months of treatment with infliximab ( $p=0.008$ ). Conclusion: By evaluating the posterior eye segment of patients with AS using OCT, this study has demonstrated that active AS patients had higher $\mathrm{ChT}$. The significant reduction in this ChT after infliximab therapy may be mediating the established effective suppressing action of infliximab on uveitis attacks.

Keywords: Ankylosing spondylitis, choroid thickness, infliximab, optical coherence tomography, retinal nerve fiber layer thickness.
\end{abstract}

Ankylosing spondylitis (AS) represents a systemic inflammatory disease affecting joints and extra-articular structures. ${ }^{1,2}$ Uveitis is among the most frequent and serious extra-articular involvements of the disease. ${ }^{3}$ Anterior uveitis findings are encountered in approximately $30-50 \%$ of cases during the course of the disease. ${ }^{1}$ However, the cause/effect-coincidence relationship between uveitis attacks and AS is still unclear, although the possibility of an association with human leukocyte antigen B27 (HLA-B27) positivity has been suggested. ${ }^{4}$ Uveitis attacks have been suggested to decrease in patients receiving anti-tumor necrosis factor-alpha (TNF- $\alpha$ ) therapy for AS. ${ }^{1}$ However, the issue of the

Received: September 17, 2019 Accepted: January 24, 2020 Published online: June 25, 2020

Correspondence: Murat Karkucak, MD. Karadeniz Teknik Üniversitesi Tıp Fakültesi Fiziksel Tıp ve Rehabilitasyon Anabilim Dalı, 61080 Trabzon, Türkiye. Tel: +90 462 - 3771048 e-mail: muratkarkucak@yahoo.com 
mechanism(s) involved in this effect has not been examined in detail.

In a previous study, we determined high choroidal thickness (ChT) in patients with AS. ${ }^{5}$ Considering the dense vascular structure and susceptibility of the choroid to inflammatory processes, ChT measurement can be used to monitor the status of systemic inflammation, particularly when ocular involvement is present.

After anti-TNF- $\alpha$ therapy, which is frequently utilized to treat AS, impairment of myelination has been proposed, without a clear mechanism. Thus, this therapy is not recommended in patients with demyelinating diseases, such as multiple sclerosis or multifocal motor neuropathy. ${ }^{1,6}$ It has been suggested that TNF- $\alpha$ may behave like a protective cytokine in demyelinating diseases in the central nervous system. The probable impacts of anti-TNF- $\alpha$ therapy on the retinal layer of subjects with AS have been investigated in a recent preliminary study (involving 21 AS patients, yet only four out of them receiving infliximab therapy, which is regarded as the firstchoice anti-TNF- $\alpha$ agent when uveitis is present), and no effects on the retinal nerve fiber layer (RNFL) thickness and ganglion cell complex (GCC) have been reported. ${ }^{6}$ For a conclusive statement on this topic, there is a need for more comprehensive clinical studies.

Hence, in this study, we aimed to quantitatively assess the profile of the ChT in patients with AS using optical coherence tomography (OCT), and to examine whether the posterior eye segment abnormalities in active AS patients are reversible by infliximab therapy.

\section{PATIENTS AND METHODS}

This study was conducted at Karadeniz Technical University Medical Faculty between October 2014 and March 2016. Thirty-one patients with AS (22 males, 9 females; mean age: $39.6 \pm 12.3$ years; range, 22 to 68 years) and 24 age- and sex-matched healthy controls (16 males, 8 females; mean age: $40.8 \pm 8.9$ years; range, 35 to 61 years) without any known systemic inflammatory disease and with normal ocular findings were included. In addition to routine examinations before and six months after anti-TNF- $\alpha$ (infliximab) therapy, choroidal and retinal examinations were performed using OCT, a novel imaging technique. The study protocol was approved by the Karadeniz Technical University Medical Faculty Ethics Committee. A written informed consent was obtained from each participant. The study was conducted in accordance with the principles of the Declaration of Helsinki.

The prior power of the study was set as $80 \%$ and $5 \%$ significance level. And, in the light of the findings obtained from our previous study on ChT in patients with AS, ${ }^{5} 25$ patients were needed. Post-hoc power analysis (using the original data from our study population of 31 patients) yielded $78.2 \%$ power (the normal approximation method) using the OpenEpi version $3.01 .^{7}$

The erythrocyte sedimentation rate (ESR), C-reactive protein (CRP), and the Bath Ankylosing Spondylitis Disease Activity Index (BASDAI) were used to assess disease activity before and after the treatment of AS patients. ${ }^{1,8}$ Infliximab, also used to treat resistant uveitis, was used as an anti-TNF- $\alpha$ agent (at a dose of $5 \mathrm{mg} / \mathrm{kg}$ ). Infliximab was given intravenously at intervals of eight weeks following the loading doses at zero, two, and four weeks. Patients in the present research were evaluated by a single physician sharing information about the study protocol.

Individuals diagnosed with AS (Modified New York Criteria 1987), meeting the conditions for the use of a biological agent (anti-TNF- $\alpha$ ) (the Assessment in Spondyloarthritis International Society criteria), and aged over 18 years were included. ${ }^{1,8}$ Subjects with eye diseases such as glaucoma, ocular hypertension, retinopathy and optic neuropathy, with a spherical equivalent refractive error higher than \pm 4.0 diopters, with a previous history of uveitis or with acute/chronic uveitis, with a history of ocular trauma and surgery or with other non-AS inflammatory diseases were excluded.

All the subjects included in the study were scanned using Optovue RTVue (RT100, Ver. A6, 7, 2, 94, Optovue, Fremont, CA, USA). The RNFL thickness, GCC, and ChT were measured. For the analysis of the RNFL thickness, "three-dimensional optic disc 
$4 \times 4 \mathrm{~mm}$ protocol" scanning was carried out. The automatic calculation of the optic nerve boundary was performed. The analysis of the optic nerve head was conducted, which consisted of 12 radial line scans with a length of $3.4 \mathrm{~mm}$ and 13 concentric rings. The device performed the automatic calculation of the RNFL thickness values acquired as a result of scanning. Furthermore, the mean GCC values were computed for all subjects using the GCC analysis (7_7 $\mathrm{mm}$ protocol).

The ChT measurement was performed as previously described. ${ }^{9}$ In brief, the scan protocol was chosen as a retina cross line that was made up of two orthogonal $6 \mathrm{~mm}$ lines, which consisted of 1024A-Scans. Consequently, for the purpose of visualizing the choroidal layer in a better way, the scan number was adjusted at 80 as a result of choosing the chorioretinal scanning mode on "Manual Tab" and the "Auto All" function on "Auto Tab". Choroidal imaging was carried out in the cross-line scanning mode. The device with manual setting measured ChT below the foveal area (Figure 1). The same researcher performed OCT for both eyes of all the subjects. Arithmetic means were computed for the OCT measurement data of both eyes, and therefore, the mean RNFL thickness, GCC, and ChT values were acquired for all subjects. Measurements performed using OCT were taken by the device in the follow mode, thus enabling identical measurements to be taken from the same point.

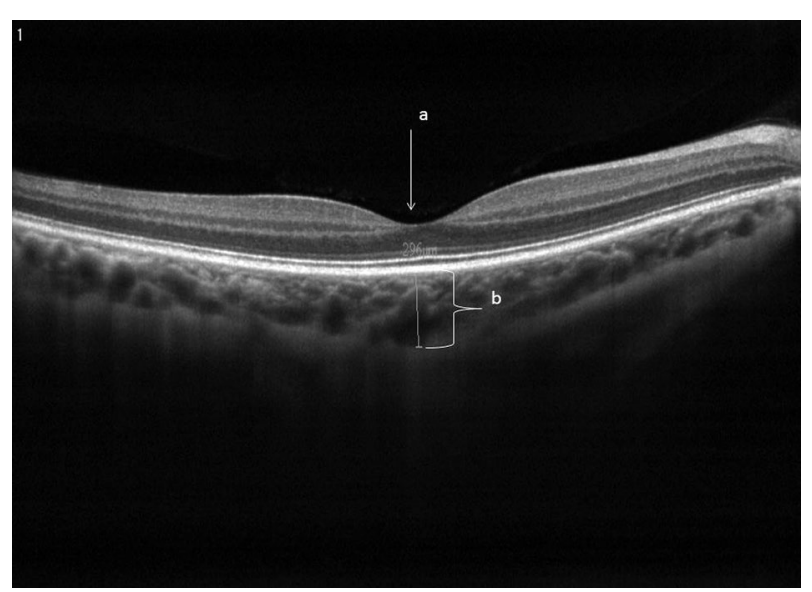

Figure 1. Image of method used for assessment of choroidal thickness on optical coherence tomography. a: Fovea; b: Measurement of choroidal thickness.

\section{Statistical analysis}

The Kolmogorov-Smirnov test was conducted for the purpose of examining the normality of distribution of data obtained by measurements. Student's t-test was carried out for comparing normally distributed measurement data between the two groups. The chi-square test was conducted for the comparison of qualitative data. Pearson's correlation analysis was applied to normally distributed data, whereas Spearman's correlation analysis was applied to non-normally distributed data. The measured data were expressed as mean \pm standard deviation, whereas data acquired by counting were presented as number (\%). Significance was adjusted at $\mathrm{p}<0.05$.

\section{RESULTS}

The two groups exhibited similarities when compared in terms of age and sex (Table 1). The CRP, ESR, and BASDAI scores of AS patients are given in Table 1 . Six months of infliximab therapy provided statistically significant improvement in the BASDAI scores in comparison with the pre-treatment values. The initial OCT parameters (ChT, RNFL thickness, and GCC) among the two groups did not exhibit significant differences (Table 2). After the therapy, significant reductions were obtained in OCT parameters, being significant for ChT and RNFL thickness, but not significant for GCC (Table 2). However, no significant change was observed in these parameters in the control group during the study period (Table 2).

Correlation analysis was conducted for the purpose of evaluating whether the posterior segment parameters reflect the status of systemic inflammation and whether infliximab therapy provides parallel changes in these parameters. As a result of the assessment, no significant correlation was determined between these parameters (BASDAI, ESR, CRP, RNFL, ChT, and GCC scores) before the treatment (RNFL vs. BASDAI $r=0.156, p=0.429$; RNFL vs. ESR $r=0.027, p=0.888$; RNFL vs. CRP $r=0.199$, $\mathrm{p}=0.292$; ChT vs. BASDAI $\mathrm{r}=0.137, \mathrm{p}=0.478$; ChT vs. ESR $r=-0.199, p=0.780$; ChT vs. CRP $r=0.141, p=0.448$ and $G C C$ vs. BASDAI $r=0.174$, $p=0.377$; GCC vs. ESR $r=-0.147, p=0.437$; GCC 
Table 1. Clinical and demographic characteristics of patients and controls

\begin{tabular}{|c|c|c|c|c|c|c|c|}
\hline \multirow[b]{2}{*}{ Parameters } & \multicolumn{3}{|c|}{ Patients with AS $(n=31)$} & \multicolumn{3}{|c|}{ Healthy controls $(n=24)$} & \multirow[b]{2}{*}{$p$} \\
\hline & $\mathrm{n}$ & $\%$ & Mean \pm SD & $\mathrm{n}$ & $\%$ & Mean \pm SD & \\
\hline Age (year) & & & $39.6 \pm 12.3$ & & & $40.8 \pm 8.9$ & 0.670 \\
\hline $\begin{array}{l}\text { Sex } \\
\quad \text { Male } \\
\text { Female }\end{array}$ & $\begin{array}{c}22 \\
9\end{array}$ & & & $\begin{array}{c}16 \\
8\end{array}$ & & & 0.479 \\
\hline Disease duration (year) & & & $7.4 \pm 3.5$ & & & - & \\
\hline \multirow[t]{2}{*}{ HLA-B27 positivity } & & 77.4 & & & - & & \\
\hline & & & Before & & & After & $p$ \\
\hline C-reactive protein $(\mathrm{mg} / \mathrm{dL})$ & & & $1.2 \pm 1.2$ & & & $1.0 \pm 0.7$ & 0.675 \\
\hline $\operatorname{ESR}(\mathrm{mm} / \mathrm{h})$ & & & $22.1 \pm 19.2$ & & & $11.5 \pm 11.2$ & 0.102 \\
\hline BASDAI & & & $6.6 \pm 1.1$ & & & $2.4 \pm 1.3$ & $0.0001^{*}$ \\
\hline
\end{tabular}

Table 2. Comparison of optical coherence tomography values of controls and ankylosing spondylitis patients treated with infliximab

\begin{tabular}{|c|c|c|c|}
\hline & Patients with AS $(n=31)$ & Healthy controls $(n=24)$ & \\
\hline & Mean \pm SD & Mean \pm SD & $p$ \\
\hline \multicolumn{4}{|c|}{ Choroidal thickness (mean) ( $\mu \mathrm{m})$} \\
\hline Before & $347.5 \pm 111.4$ & $322.1 \pm 62.8$ & 0.283 \\
\hline After & $326.8 \pm 106.9$ & $322.2 \pm 59.9$ & 0.858 \\
\hline$p$ & $0.018^{*}$ & 0.920 & \\
\hline \multicolumn{4}{|c|}{ Retinal nerve fiber layer (mean) $(\mu \mathrm{m})$} \\
\hline Before & $106.4 \pm 13.5$ & $104.4 \pm 9.1$ & 0.643 \\
\hline After & $105.0 \pm 13.0$ & $103.5 \pm 9.1$ & 0.773 \\
\hline$p$ & $0.008^{*}$ & 0.102 & \\
\hline \multicolumn{4}{|c|}{ Ganglion cell complex (mean) ( $\mu \mathrm{m})$} \\
\hline Before & $97.0 \pm 8.7$ & $98.0 \pm 5.4$ & 0.613 \\
\hline After & $96.6 \pm 9.7$ & $98.6 \pm 6.0$ & 0.452 \\
\hline$p$ & 0.375 & 0.176 & \\
\hline
\end{tabular}

vs. CRP $\mathrm{r}=0.184, \mathrm{p}=0.330$ ). However, after infliximab treatment, some parameters became significant (RNFL vs. BASDAI $\mathrm{r}=0.133, \mathrm{p}=0.464$; RNFL vs. ESR $r=0.679, p=0.004$; RNFL vs. CRP $\mathrm{r}=0.160, \mathrm{p}=0.500$; ChT vs. BASDAI $\mathrm{r}=-0.703$, $p=0.751 ; \quad$ ChT vs. ESR $r=0.125, p=0.644$; ChT vs. CRP $r=0.234, p=0.308$ and GCC vs. BASDAI $r=0.703, p=0.377$; $G C C$ vs. ESR $r=0.688, p=0.003 ;$ GCC vs. CRP $r=0.703$, $\mathrm{p}=0.931$ ).

\section{DISCUSSION}

The current research examines the impact of infliximab, an anti-TNF- $\alpha$ agent preferably used in AS with uveitis attacks, on ChT in patients with AS without any evident current uveitis or a history of uveitis. We found out that six-months of infliximab therapy reduces ChT. This finding indicates that vascular structural changes in the posterior ocular segment in AS are reversible. 
Ocular involvement in AS generally manifests itself in the form of anterior uveitis, and the incidence of this involvement increases with HLA-B27 positivity. ${ }^{3}$ In uveitis associated with HLA-B27 positivity, the posterior segment as well as the anterior segment has been shown to be affected. Balaskas et al. ${ }^{4}$ determined that retinal thickness increased in line with inflammation in the course of an HLA-B27-associated anterior uveitis attack and that the mentioned increase declined in a gradual way as the attack was resolved by therapy with topical corticosteroids. Following this study, which revealed the secondary retinal effects caused by inflammation in the uveal tissue, the researchers focused on the choroid, the tissue prone to be affected by inflammation. In a recent OCT study, we determined greater ChT in patients with AS even without uveitis attacks compared to the healthy population. ${ }^{5}$ This finding, identified for the first time in the literature, tempts to presume that ChT could be an early indicator of ocular involvement in AS, particularly when there are no evident uveitis attacks.

The use of OCT has become increasingly widespread in ophthalmology. This technique permits noninvasive and objective sectional imaging of tissues, such as the choroid, cornea, and retina. Several studies have reported that the technique provides highly repeatable and sensitive measurements at the micron level. ${ }^{10-13}$ Corneal thickness, papillary RNFL, GCC, foveal thickness, and ChT measurements obtained using OCT are widely used nowadays in the diagnosis and monitoring of different eye diseases. ${ }^{10-13}$

In a cross-sectional study, Ayhan Tuzcu et al. ${ }^{14}$ detected no statistically significant difference in peripapillary RNFL thickness in AS patients in comparison with the control group. Nevertheless, in the subgroup analysis of the patients, they reported lower peripapillary RNFL thickness in subjects having high disease activity and thinning of the ganglion cell layer. ${ }^{14}$ Kim et al. ${ }^{15}$ determined increased ChT in the development of Behçet's disease-related active posterior uveitis, and they identified that this increase then declined as uveitis improved with therapy. This study also reported that there was a correlation between decreased ChT and a decrease in retinal vessel leakage in fundus fluorescein angiography. ${ }^{15}$ In another study, Ataş et al. ${ }^{16}$ reported greater ChT in patients with Behçet's disease. We could not directly compare our results with any report in the literature, but the thickening of ChT in systemic inflammatory diseases with eye involvement is consistent with our findings obtained from the present study. ${ }^{14-16}$

Anti-TNF- $\alpha$ therapy is commonly utilized across the world to treat AS. Despite the high cost of this treatment, it is very effective since it does not only provide positive effects on inflammation but also reduces pain and increases functional capacity in AS. The number of studies that investigate the effect of anti-TNF- $\alpha$ on extraarticular involvement is increasing. ${ }^{17-19}$ Biological agent therapies are also used to treat uveitis associated with AS. Placebo-controlled studies with one of these therapies, etanercept, have not demonstrated any effectiveness in the treatment of uveitis, and etanercept is not regarded nowadays as the first-choice agent in the treatment of uveitis due to reports of new attacks in patients under treatment for rheumatologic diseases. Infliximab is more commonly used in the treatment of acute uveitis attacks because of its rapid and powerful effects reported by clinical studies. Adalimumab is preferred in chronic childhood uveitis due to the ease of administration and the low risk of allergic reactions. In a recent review, infliximab and adalimumab were recommended as a second-line therapy in the case of severe ocular inflammation. ${ }^{20}$ Therefore, in this pioneering study, we preferred infliximab among anti-TNF- $\alpha$ agents for investigating the possible effects on AS-related ChT changes.

In a recent study, probable impacts of antiTNF- $\alpha$ therapy on the retinal layer of subjects with AS were assessed. This study is rather preliminary because it involved only 21 AS patients and yet only four of these patients received infliximab. No effect on nerve cells in the retinal layer thickness was reported. 6 In addition to RNFL thickness, we also measured ChT and analyzed the effects of infliximab in 31 patients and determined that retinal cells were also affected by the therapy. The discrepancy between our findings and the findings obtained by Ilhan et al. ${ }^{6}$ could be due to the fact that their patient population was small and the therapy agents were heterogeneous. This is striking considering the negative effect of etanercept (10 out of 21 patients received this agent) in this regard, which has been mentioned above. Our study also involved a control group 
(the absence of statistically significant changes also indicates that the obtained effect is not a time-dependent change), which does not exist in the latest study. Considering the physiological role of the choroid in relation to the neighboring structures, including the retina, the observed changes could be secondary to changes in the choroid layer obtained by the therapy.

The probable reason for uveitis attacks being less common in subjects receiving antiTNF- $\alpha$ therapy may be that uveal tissues, and thus also the choroid, are less affected. The effective suppression of inflammation can also lead to abolishment of responses secondary to inflammation. The result may be less inflammatory cell migration and less choroidal vessel leakage, and therefore, a lower increase in the choroid layer thickness.

Our study has some limitations. Firstly, the six-month therapy, based on the clinical status of AS, is effective while structural changes in the posterior eye segment may take a longer duration and may not be reflected adequately when these parameters are evaluated only six months after the therapy. Secondly, we found only a mild-level correlation between the disease activity parameters and investigated posterior eye segment parameters posing the rationale of the investigation to question. However, since we measured the disease activity parameters from the systemic blood, they could not reflect the local inflammatory status in the eye.

In conclusion, using OCT, we quantitatively assessed the profile of ChT in patients with AS and found that, although not significant compared to the controls, AS patients had higher ChT and the six-month standard therapy targeted for AS with infliximab effectively reduced ChT and RNLF in these patients. The results obtained from this study indicate that posterior eye segment changes associated with acute anterior uveitis attacks, the eye involvements occurring in approximately $30-40 \%$ of subjects with AS during the course of the disease, are reversible by infliximab therapy. These results could be extrapolated as the mentioned ChT-decreasing impact of infliximab can be included in its efficient suppressing mechanism of uveitis attacks not only in AS but also in chronic uveitis cases, refractory to conventional therapy.

\section{Declaration of conflicting interests}

The authors declared no conflicts of interest with respect to the authorship and/or publication of this article.

\section{Funding}

The authors received no financial support for the research and/or authorship of this article.

\section{REFERENCES}

1. Braun J, van den Berg R, Baraliakos X, Boehm H, Burgos-Vargas R, Collantes-Estevez E, et al. 2010 update of the ASAS/EULAR recommendations for the management of ankylosing spondylitis. Ann Rheum Dis 2011;70:896-904.

2. Karkucak M, Çakırbay H, Capkın E, Topbas M, Guler $\mathrm{M}$, Tosun $\mathrm{M}$ et al. The prevalence of ankylosing spondylitis in the eastern Black Sea region of Turkey. Eur J Gen Med 2010;8:40-5.

3. Elewaut D, Matucci-Cerinic M. Treatment of ankylosing spondylitis and extra-articular manifestations in everyday rheumatology practice. Rheumatology 2009;48:1029-35.

4. Balaskas K, Ballabeni P, Guex-Crosier Y. Retinal thickening in HLA-B27-associated acute anterior uveitis: evolution with time and association with severity of inflammatory activity. Invest Ophthalmol Vis Sci 2012;53:6171-7.

5. Kola M, Kalkisim A, Karkucak M, Turk A, Capkin E, Can I, et al. Regarding Comments by Yolcu et al. on "Evaluation of Choroidal Thickness in Ankylosing Spondylitis Using Optical Coherence Tomography". Ocul Immunol Inflamm 2015;23:465-6.

6. Ilhan N, Ustun N, Tuzcu EA, Coskun M, Yagiz AE, Ilhan $\mathrm{O}$, et al. Spectral domain-optical coherence tomographic findings in patients with ankylosing spondylitis under anti-tumor necrosis factor-alpha therapy. Cutan Ocul Toxicol 2015;34:222-6.

7. Dean AG, Sullivan KM, Soe MM. Open Epi:Open Source Epidemiologic Statistics for Public Health. Available at: http://www.openepi.com/Menu/OE_ Menu.htm updated 2013/04/06, 2014/06/26

8. van der Linden S, Valkenburg HA, Cats A. Evaluation of diagnostic criteria for ankylosing spondylitis. A proposal for modification of the New York criteria. Arthritis Rheum 1984;27:361-8.

9. Branchini L, Regatieri CV, Flores-Moreno I, Baumann B, Fujimoto JG, Duker JS. Reproducibility of choroidal thickness measurements across three spectral domain optical coherence tomography systems. Ophthalmology 2012;119:119-23.

10. Moseng L, Sæeter M, Mørch-Johnsen GH, Hoff JM, Gajda A, Brodtkorb E, et al. Retinal nerve fibre layer attenuation: clinical indicator for vigabatrin toxicity. Acta Ophthalmol 2011;89:452-8. 
11. Adhi M, Duker JS. Optical coherence tomography-current and future applications. Curr Opin Ophthalmol 2013;24:213-21.

12. Turk A, Ceylan OM, Arici C, Keskin S, Erdurman $\mathrm{C}$, Durukan $\mathrm{AH}$, et al. Evaluation of the nerve fiber layer and macula in the eyes of healthy children using spectral-domain optical coherence tomography. Am J Ophthalmol 2012;153:552-9.

13. Branchini LA, Adhi M, Regatieri CV, Nandakumar $\mathrm{N}$, Liu JJ, Laver $\mathrm{N}$, et al. Analysis of choroidal morphologic features and vasculature in healthy eyes using spectral-domain optical coherence tomography. Ophthalmology 2013;120:1901-8.

14. Ayhan Tuzcu E, Ustun N, Ilhan N, Yagiz E, Daglioglu MC, Coskun M, et al. Peripapillary retinal nerve fiber layer and ganglion cell-inner plexiform layers thickness in ankylosing spondylitis. Ocul Immunol Inflamm 2014;22:429-33.

15. Kim M, Kim H, Kwon HJ, Kim SS, Koh HJ, Lee SC. Choroidal thickness in Behcet's uveitis: an enhanced depth imaging-optical coherence tomography and its association with angiographic changes. Invest Ophthalmol Vis Sci 2013;54:6033-9.
16. Ataş M, Yuvacı I, Demircan S, Güler E, Altunel O, Pangal E, et al. Evaluation of the Macular, Peripapillary Nerve Fiber Layer and Choroid Thickness Changes in Behçet's Disease with Spectral-Domain OCT. J Ophthalmol 2014;2014:865394.

17. Capkin E, Kiris A, Karkucak M, Durmus I, Gokmen F, Cansu A, et al. Investigation of effects of different treatment modalities on structural and functional vessel wall properties in patients with ankylosing spondylitis. Joint Bone Spine 2011;78:378-82.

18. Capkin E, Karkucak M, Kiris A, Durmus I, Karaman $\mathrm{K}$, Karaca A, et al. Anti-TNF-alpha therapy may not improve arterial stiffness in patients with AS: a 24-week follow-up. Rheumatology 2012;51:910-4.

19. Karkucak M, Capkın E, Kırıs A, Sahin M, Kücükali A, Karaca A et al. Anti TNF-alpha therapy and arterial stiffness in ankylosing spondylitis; results with long-term 2 year follow-up. Arch Rheumatol 2014;29:250-6.

20. Levy-Clarke G, Jabs DA, Read RW, Rosenbaum JT, VitaleA, Van GelderRN.Expertpanel recommendations for the use of anti-tumor necrosis factor biologic agents in patients with ocular inflammatory disorders. Ophthalmology 2014;121:785-96. 